\title{
The mechanobiology of craniosynostosis in Crouzon syndrome
}

Mehran Moazen $^{\mathrm{a} \dagger}$, Mahbubeh Hejazi ${ }^{\mathrm{a} \dagger}$, Dawn Savery ${ }^{\mathrm{b}}$, Dominic Jones ${ }^{\mathrm{c}}$, Arsalan Marghoub $^{\mathrm{a}}$, Ali Alazmanic, Erwin Pauws ${ }^{\mathrm{b}^{*}}$

${ }^{a}$ UCL Mechanical Engineering, University College London, London, United Kingdom

${ }^{\mathrm{b}}$ Developmental Biology \& Cancer Research and Teaching Department, UCL Great Ormond Street Institute of Child Health, University College London, London, United Kingdom

${ }^{c}$ School of Mechanical Engineering, University of Leeds, Leeds, United Kingdom

${ }^{\dagger}$ These authors contributed equally.

*Corresponding author: e.pauws@ucl.ac.uk

\section{Highlights}

- Mechanical loading of calvarial bone suspends craniosynostosis.

- Loading effects on cranial base reduce midfacial hypoplasia.

\section{Key words}

craniosynostosis, mechanobiology, bone, suture, FGFR2, Crouzon 


\section{Abstract}

2 Children with syndromic forms of craniosynostosis undergo a plethora of surgical interventions to resolve the

3 clinical features caused by the premature fusion of cranial sutures. While surgical correction is reliable, the

4 need for repeated rounds of invasive treatment puts a heavy burden on the child and their family.

5 This study explores a non-surgical alternative using mechanical loading of the cranial bones to prevent or 6 delay craniosynostosis and its effects.

7 We treated Crouzon syndrome mice before the onset of craniosynostosis by mechanical loading of cranial 8 bones.

9 Cranial loading applied to the frontal bone caused partial normalisation of skull shape, reducing the typical

10 brachycephalic appearance. This is underpinned by the delayed closure of the coronal suture and of the

11 intersphenoidal synchondrosis.

12 This study provides a novel treatment alternative for syndromic craniosynostosis which has the potential to be

13 an important step towards replacing, reducing or refining the surgical treatment of craniosynostosis patients. 


\section{Introduction}

22 Some of the most common birth defects affect the craniofacial development of a new-born baby. Therefore, the care of children with craniofacial birth defects is an important clinical activity. Diagnosis and treatment of the disorders typically involves a child being seen by a number of specialists in areas including clinical genetics, radiology, dentistry, maxillo-facial and neurosurgery, and anaesthesia. Craniosynostosis is a common birth defect with an estimated prevalence between 1 in $2,100^{1}$ and 1 in 4,200 incidence ${ }^{2}$. It is caused by the premature fusion of the bones that form the skull, which can have serious, possibly life-threatening results $^{3,4}$. Traditional approaches to the treatment of this type of craniofacial birth defect employ complex surgical remodelling of the skull including facial deformities, aimed at protecting brain development, visual function and the restoration of a more normal craniofacial appearance. While the surgical correction of the craniofacial deformity and its secondary defects is reliable in many cases, syndromic forms of craniosynostosis are often more severe and there is a heavy burden of care and ongoing surgical intervention from birth to maturity.

The most severe forms of craniosynostosis that require the most intensive treatments are often syndromic and single-gene disorders 5 . While the underlying pathogenesis of syndromic cases of craniosynostosis is complex and can involve a plethora of developmental signalling pathways, the majority of cases are caused by mutations in genes directly or indirectly involved in the FGF signalling pathway ${ }^{6}$. In addition, abberant FGF signalling plays an important role in many other developmental skeletal defects, affecting endochondral as well as intramembranous ossification ${ }^{7}$. Besides abnormalities in cellular physiology, studies showing an association between intrauterine constraint and increased incidence of craniosynostosis indicate a role for external mechanical forces on the formation and/or homeostasis of cranial sutures ${ }^{8}$.

42 Surgical treatment of syndromic craniosynostosis can be clinically challenging because in many cases multiple sutures are affected. Also, the genetic and phenotypic variability between patients, even those within the same syndrome classification is often significant enough to require individualised treatment protocols ${ }^{9,10}$. In addition, these patients require an increased number of craniofacial procedures ${ }^{5}$. The number of postsurgical complications is higher in syndromic cases, with increased rates seen in secondary synostosis ${ }^{11}$. There

47 is also a high number of complication associated with frontonasal distraction procedures ${ }^{12}$. 
Despite the rapid evolution of human genetics, gene therapy and stem cell therapy during the last few decades, very few studies have been able to find suitable alternatives to surgery. While our understanding of the pathogenesis of syndromic craniosynostosis has increased significantly -mainly through the use of genetically manipulated mouse models- this has not resulted in any clinically applicable non-surgical treatment alternatives ${ }^{13}$. To date, some of the most promising studies have tried to identify a biological solution to the treatment or prevention of craniosynostosis using gene therapy or drug development. Using transgenically modified mouse strains, attempts have been made to use either RNA interference against a mutation causing Apert syndrome, knockout of PIN1 in an Apert model or overexpression of the FGFR2c isoform to target a mutation causing Crouzon syndrome ${ }^{14-16}$. All successfully improved the synostosis phenotype in vivo, but to date have not been translated into clinical application. Pharmacologically, a host of studies have tried to identify drug targets that can possibly delay or prevent craniosynostsis in a mouse model. A drug screen of 640 FDA approved drugs identified 14 small molecule inhibitors that affect normal craniofacial development in zebrafish and went on to show that one of these prevented craniosynostosis in a mouse model for Crouzon syndrome ${ }^{17}$. Another study was able to use viral delivery of tissue nonspecific alkaline phosphatase (TNAP) to partially rescue the craniosynostosis phenotype in a Crouzon mouse model ${ }^{18}$. Finally, the recent study by Yu et al. is the only example so far of in vivo suture regeneration using stem cells that has the potential for clinical application ${ }^{19}$. Mechanotransduction is the process whereby cells convert mechanical stimulus into a molecular and cellular response. While there is a large body of literature on mechanical loading of endochondral bone ${ }^{20}$, only recently studies have started to explore how intramembranous, craniofacial bones and sutures respond to mechanical forces. These studies have shown that craniofacial sutures respond to cyclical, mechanical loading using both in vitro and in vivo models. Resected calvaria of wild-type rat skulls were loaded on the posterior intrafrontal suture under tension in an ex vivo model. It was reported that loaded sutures remained patent while in the control group the suture fused normally ${ }^{21}$. In vivo cyclical loading applied to the skull (i.e. maxilla) has been shown to increase the width of the premaxillomaxillary suture by approximately $30 \%$ compared to controls in rat $^{22}$ and similar results have been obtained in rabbit ${ }^{23}$. Normal craniofacial sutures in 
cells, while compression forces lead to increased osteogenesis ${ }^{24}$. Therefore, the aforementioned studies suggest that cyclical, mechanical loading can keep cranial sutures patent during postnatal development and delay their natural closure.

Many mouse models have been developed that feature the craniosynostosis phenotype ${ }^{13}$. For this study we used an established model of Crouzon syndrome. Crouzon mice carry a mutation (p.C342Y) in the FGFR2 receptor, are viable and fertile and are characterised by brachycephaly caused by coronal craniosynostosis as well as midfacial hypoplasia associated with malocclusion ${ }^{25}$. Our laboratory has been studying this mouse model since 2010 and have built up considerable knowledge of the cellular and molecular events underlying the pathogenesis of the phenotypic features this animal model exhibits, including impaired mesenchymal condensation of skeletal elements due to misregulation of $\mathrm{SOX}^{26}$. A study from Hatch et al. showed that genetic background effects phenotypic severity with a more pronounced craniofacial phenotype in C57BL/6 mice compared to $\mathrm{BALB} / \mathrm{c}^{27}$. Our mice have been backcrossed onto the $\mathrm{CD}-1$ strain, which mimicks human Crouzon syndrome and on our hands displays synostosis of the coronal suture at three weeks of age (P21). We have also previously shown differences in biomechanical properties of individual calvarial bones (i.e. frontal bone vs parietal bone) in Crouzon syndrome calvarial bones and sutures ${ }^{28}$. These observations point to a fundamental difference between the architecture of normal craniofacial bone and suture tissue affected by FGFR2 mutation. These observations are supported by studies showing that the neural crest derived frontal bone is more susceptive to FGFR2 perturbation than the mesoderm derived parietal bone ${ }^{15,29}$.

93 This study aims to investigate the possibility that external mechanical force applied to cranial bone can delay 94 or prevent closure of abnormal sutures prone to craniosynostosis. Previous research has shown that 95 mechanical loading of cranial bone in can delay the normal closure of cranial sutures during postnatal 96 development. Our hypothesis is that in vivo mechanical loading of calvarial bone can significantly delay 97 premature, pathogenic suture closure in a mouse model for craniosynostosis. 


\section{Results}

In vivo mechanical loading of cranial bone in a Crouzon syndrome mouse model.

To test our hypothesis that mechanical loading of the skull can prevent pathogenic suture closure, we performed in vivo loading of cranial bones on pre-weaning Crouzon mice. We developed a bespoke system using a linear actuator and force sensor enabling us to carry out controlled and repeatable loading of the cranial bones (Figure 1a). The actuator moved a force sensor in the dorso-ventral direction while a probe was fixed to it, in effect loading the skull via the probe. The loading tip of the probe that came into contact with the skull had a flat circular surface with a diameter of $1 \mathrm{~mm}$. Based on previous work on rabbit and rat models accurate, positional loading on cranial bones of pre-weaning mice, we used general aneasthesia by Isoflurane inhalation to fix animals in position using a custom-made, 3D printed tube underneath the actuator probe for the duration of the loading procedure (Figures 1B and 1C). We loaded all animals on the left side of the cranium to allow us to compare with the right side as an unloaded control (Figure 1D). Because of the overlapping nature of the coronal suture (Figure 1E) we decided to perform loading experiments on the frontal and parietal bone seperately. This way, we were able to compare the application of tension force on the suture when loading on the frontal bone and compression force on the suture when loading on the parietal bone (Figure 1F). We used an Fgfr2-C342Y transgenic mouse line that is kept on a CD-1 background, that in our hands displays patent sutures at birth, followed by the onset of craniosynostosis which is usually complete by P21. To maximise the potential impact of our cranial bone loading treatment, we loaded animals for 10 days between P7-18. Skulls were subsequently dissected and analysed at P21 (Figure 1G). Supplementary

119 Videos 1a and 1b show examples of the cranial bone loading procedure at P7 and P14 respectively. In all 120 experiments, mice recovered quickly after daily cycles of aneasthesia and cranial bone loading and at P21, 121 after 10 rounds of treatment, animals appeared healthy (Supplementary Video 2a and b). 

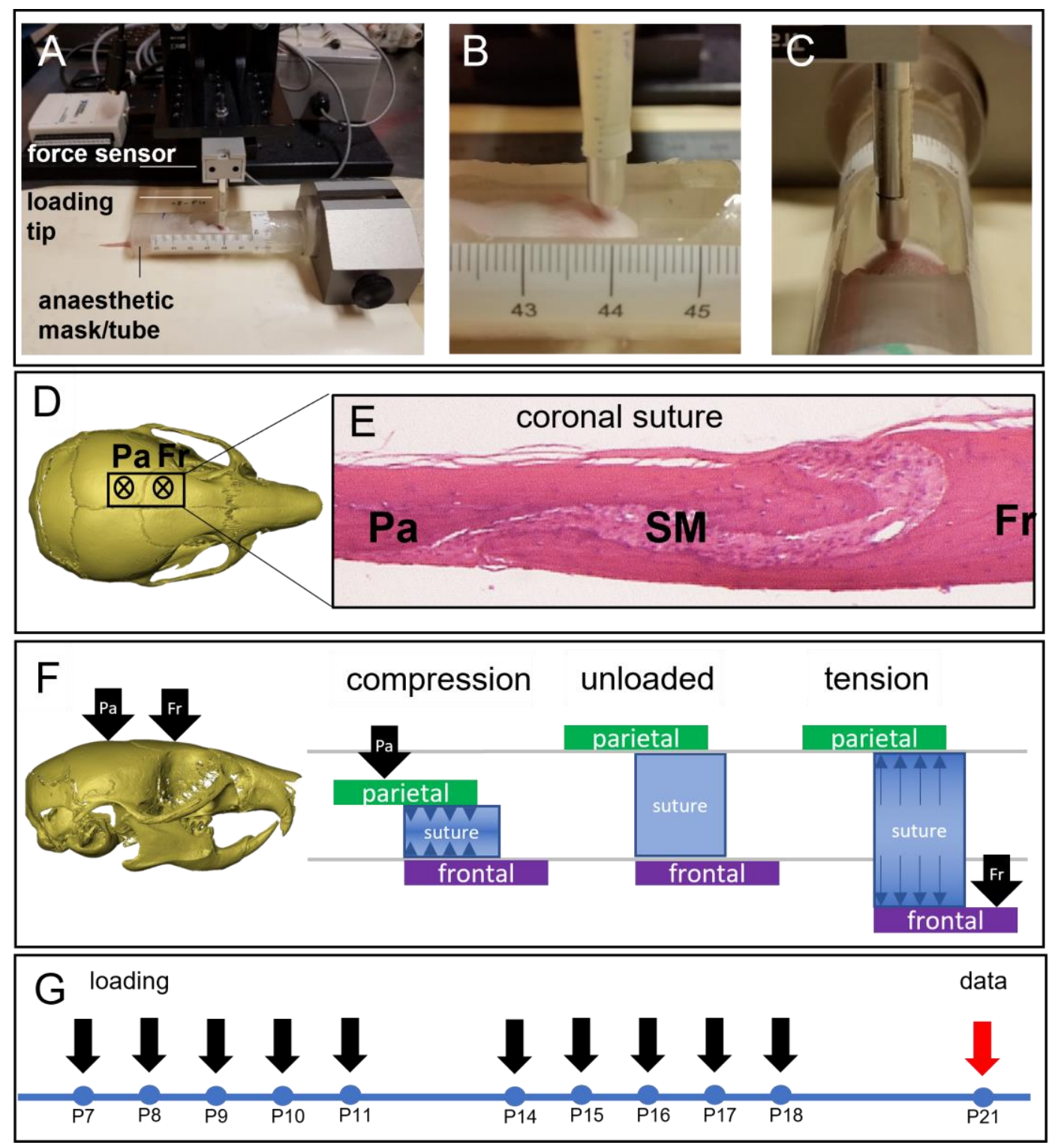

Figure 1

Cranial bone loading on pre-weaning Crouzon mice using actuator technology.

A Equipment set-up showing P10 mouse in a custom-designed, 3D-printed anaesthetic mask and holder, with a window for access to the skull vault. The electrical, linear actuator moves a small probe $(1 \mathrm{~mm})$ that loads the bone underneath, a sensor records the force applied. B, C Close-ups of the loading protocol in progress at postnatal day 7 (B) and 21 (C) with the actuator probe targeting the frontal bone. D Top view of a micro-CT generated image of a wild-type mouse skull (P21). The asterisks indicate the approximate loading locations on the parietal (Pa) and frontal (Fr) bones, either side of the coronal suture. E Histological, sagittal section (H\&E) through an adult mouse skull showing the parietal (Pa) and frontal (Fr) bones and the suture mesenchyme (SM) situated between the overlapping parts of the parietal and frontal bones. F Schematic representation of the mechanical action generated by loading on either the parietal (Pa) or frontal (Fr) bones. Loading the frontal bone will generate tensile forces on the suture (right) and loading the parietal bone will generate compression forces on the suture (left). G Treatment schedule for the cranial loading protocol starting at P7 and loading 10 days until P18. Skulls are collected at P21.

\section{Changes in Crouzon skull morphology after cranial bone loading.}


138 We applied cyclical, mechanical loading to the frontal bone of wild-type (WT; n=15) and FGFR2-C342Y 139 (MUT; $n=7)$ mice and to the parietal bone of WT $(n=9)$ and MUT $(n=8)$ mice for 10 days between P7 and 140 P18. On the last day of loading, mice were injected with Alizarin complexone and on P21 mice were culled and skulls dissected. Skulls were then microCT scanned for further morphological characterisation. In all our subsequent analyses, we compared treated mice with untreated control mice, WT $(n=12)$ and MUT $(n=16)$, at

143 P21. In the untreated group (Figure 2A), we could clearly see the typical phenotypic appearance of the 144 Crouzon mouse demonstrated by a domed, brachycephalic skull vault and a shortened maxilla which in the most severe cases resulted in malocclusion of the incisors. Superfical analysis of the surface of the skull vault showed complete synostosis of the coronal suture. Analysis of the mutants loaded on the frontal bone showed a distinctive shift to a more normal skull appearance, with reduced doming of the skull and elongated maxillary area (Figure 2B). Strikingly, the coronal suture was still absent in most cases at P21. Mice loaded on the parietal bone showed no improvement of Crouzon-realted appearance (Figure 2C). No apparent effect was noted on WT loaded skulls. To quantify these gross morphological observations, we performed detailed analysis of skull morphologies (i.e. length, width and height) using the microCT images (Figures 2D-E).

$(\Delta 12.2 \%, \mathrm{p}=9.2 \mathrm{E}-11)$, height $(\Delta 13.4 \%, \mathrm{p}=2.1 \mathrm{E}-12)$ when compared to the untreated WTs, represented by the phenotypic effects of midfacial hypoplasia and brachycephaly. Comparing the skull morphologies of the treated and untreated mutant mice, showed distinct differences suggesting phenotypic rescue, but only in the group treated on the frontal bone. Again, no difference was noted between treated and untreated WT skulls.

The dimensions of the untreated mutant skull were used as control in all subsequent comparisons to compare the effect of the treatment. Mice treated on the frontal bone showed a statistically significant reduction of the maxillary shortening compared to untreated mutants (skull length: $\Delta 5.9 \%$ vs $\Delta 12.2 \%, \mathrm{p}=2.9 \mathrm{E}-04$ ).

Brachycephalic appearance measured by the skull height was reduced in frontal loaded skulls $(\Delta 10.7 \%$ vs 

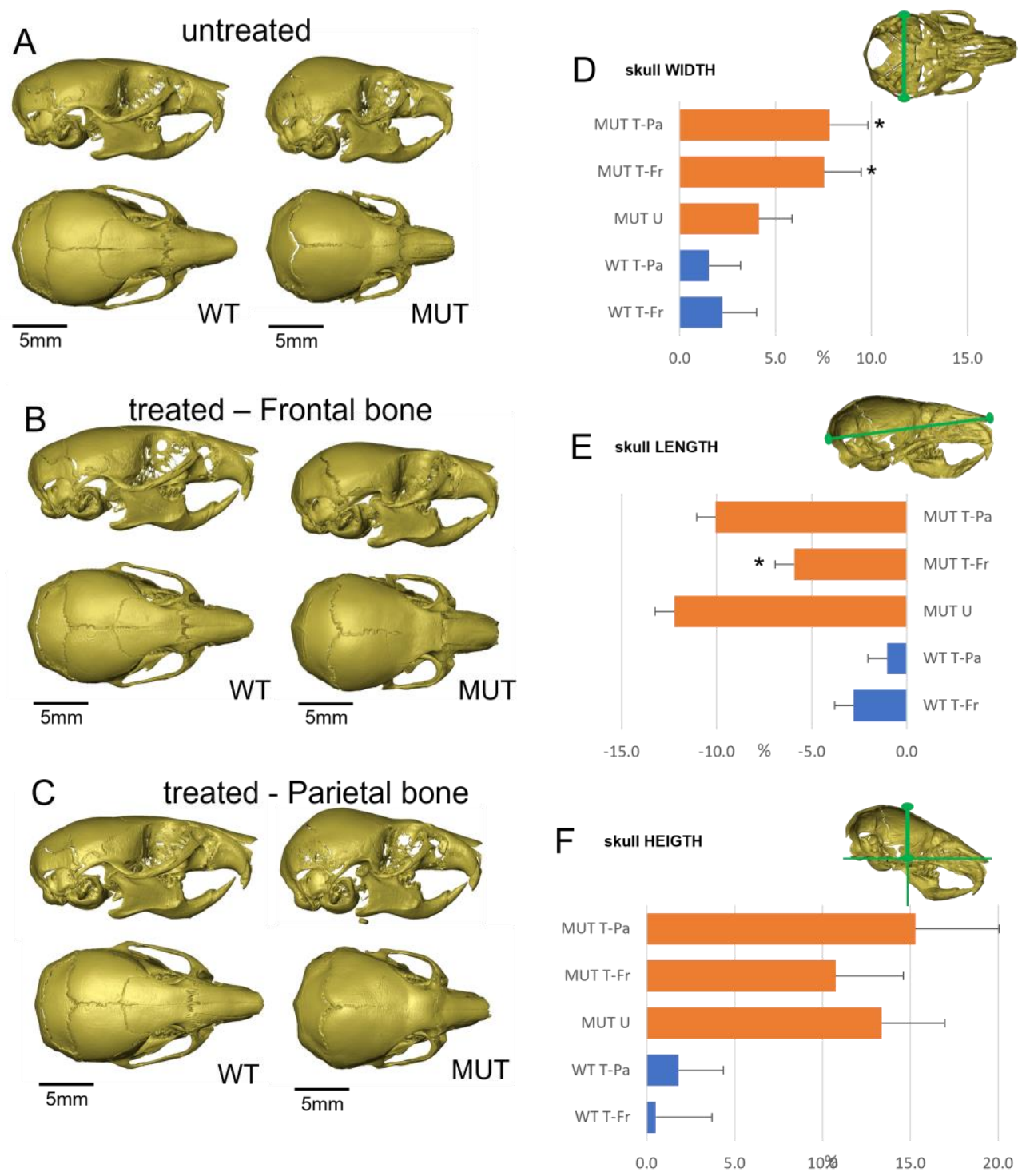

Figure 2 Changes in Crouzon skull morphology after cranial bone loading.

A micro-CT images of untreated P21 skulls. MUT (Fgfr2_C342Y) skulls show typical signs of brachycephaly and synostosis of the coronal suture. B micro-CT images P21 skulls loaded on the frontal bone. While WT skulls appear unchanged, MUT skulls display a normalised skull shape with a reduced brachycephalic appearance. Synostosis of the coronal suture does not appear to be affected. C micro-CT images P21 skulls loaded on the parietal bone. Compared to untreated skulls, no changes can be observed. D Relative dimensions -in percentage increase/decrease- compared to untreated WT skulls show that frontal and parietal loading increases mutant skull width by respectively $7.5 \%$ and $7.8 \%$ as compared to $4.1 \%$ in untreated mutant skulls. E Untreated, mutant skulls are $12.2 \%$ shorter than untreated WT skulls, indicating the midfacial hypoplasia phenotype. Only frontal bone loaded skulls show a statistically significant difference and are only 5.9\% shorter. F Untreated, mutant skulls are 13.4\% higher than untreated WT skulls, indicating the brachycephalic appearance of these skulls. No statistically significant differences are found for relative height in treated skulls. Images in A-C are representative of the average dimensions per experimental group. Untreated-WT $n=12$, frontal loaded-WT (WT T-Fr) n=15, parietal loaded-WT (WT T-Pa) n=9, untreated MUT (MUT U) $n=16$, frontal loaded MUT (MUT T-Fr) n=7, parietal loaded MUT (MUT T-Pa) $n=8 . *=$ statistically significant $(\mathrm{p}<0.05)$ based on T-test performed against MUT U. Error bars in D-F represent approximate variance calculated using the Delta method. 
In an attempt to explain the gross phenotypic effects on skull size and shape after cranial bone loading, we

of the suture (Figure 3B). Skulls loaded on the frontal bone showed calvaria with patent sutures in most cases, way we observed that some skulls exhibit an asymmetrical partial rescue of coronal synostosis on the treated (left) side. Other skulls show a wider effect where both sutures remained patent at P21. When using an unbiased (i.e. randomised and blinded) suture patency scoring system, loaded sutures were more likely to be patent or only partially fused then controls. This effect was larger on the treated side $\left(X^{2}=9.61, p=0.0019\right)$ than on the untreated side $\left(\mathrm{X}^{2}=4.23, \mathrm{p}=0.0397\right)$. Histological data confirmed the morphological observations. Untreated WT sutures displayed the typical overlapping structure, with the parietal bone on top of the frontal bone and the sutural mesenchyme in between (Figure 3F). Untreated mutant sutures had lost the normal, patent structure and instead showed scattered areas of mesenchyme surrounded by a larger amount of bone (Figure 3G). Sutures loaded under tension (frontal bone) had increased patency, but also showed increased same histological preparations we analysed the brain tissue underneath the location of loading to exclude any secondary effects on the underlying cortex. In none of the samples analysed, we observed any signs of abnormal cortical lamination (Supplementary Figure 1). Therefore, we can conclude that cranial loading on the frontal bone, inducing tension on the suture, causes partial rescue in the majority of cases and total rescue in a minority of cases without any damage to the underlying brain. 

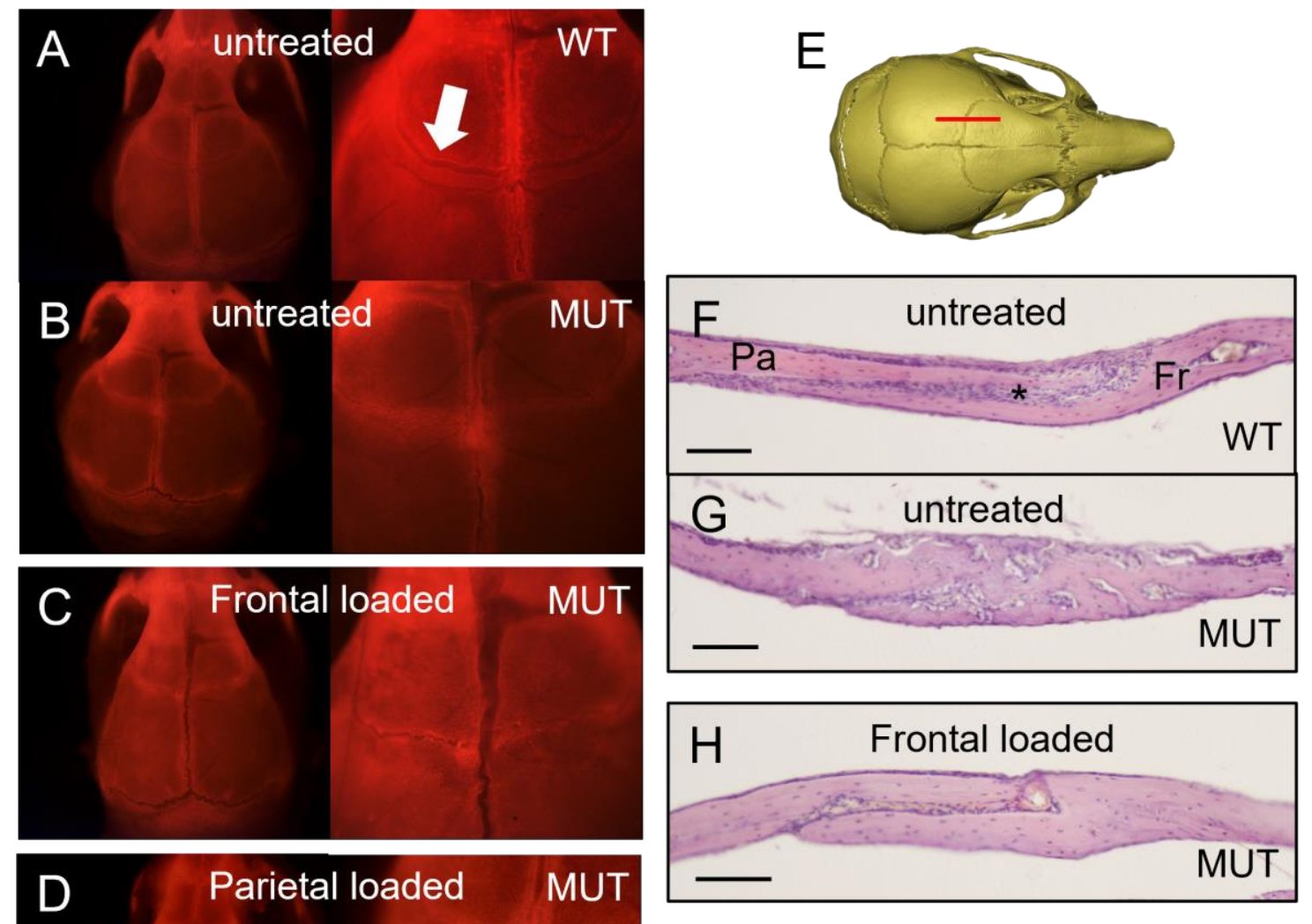

Figure 3

Changes in coronal suture morphology and histology following cranial bone loading.

A-D Calvaria showing fluorescent Alizarin complexone staining at 0.8x (left) and 2x (right) magnification. A Wild-type (WT) calvaria show the typical overlapping bone structure of the coronal suture (arrow). B Mutant (MUT) coronal sutures show some staining indicating active bone growth but the overlapping structure is absent. C Frontal loaded calvaria show a patent suture, but lack the overlapping structure. D Parietal loaded calvaria show no difference from untreated mutant sutures. E Sagittal sections through the coronal suture (red line) at P21 were stained with H\&E. F Wildtype (WT) sutures show a characteristic overlapping feature of the parietal $(\mathrm{P})$ and frontal $(\mathrm{F})$ bones with the suture mesenchyme (*) in between. G Mutant (MUT) sutures show a loss of patency phenotype with fusion between parietal and frontal bones and loss of mesenchyme. The suture appears thickened as compared to flanking bone. H Animals treated with frontal bone loading show a more normal suture with bones appearing unfused, but with significant loss of mesenchyme. I Animals treated with parietal bone loading show no improvement and look similar to untreated sutures. Scale bars are $100 \mu \mathrm{m}$.

221 Changes in skull base morphology and histology following cranial bone loading.

222 Analysis of the gross skull morphology showed that mutant mice loaded on the frontal bone display a reduced 223 maxillary shortening (Figure 2E). While the delayed synostosis of the coronal suture may contribute to this, 
midline through the cranial base to assess the morphology. At P21, both the sphenoidal-occipital

226 synchondrosis (SOS) and the intersphenoidal synchondrosis (ISS) were open in WT mice, but in the untreated

227 mutants, the ISS is partially or fully closed (Figure 4B). This contributes to the maxillary reduction and the

228 resulting midfacial hypoplasia seen in Crouzon mice. The majority of mutant mice loaded on the frontal bone

229 had a partially open ISS, sometimes with a bony bridge located on the caudal side of the cranial base (Figure

230 4C). Parietal bone loaded mutants showed no difference to untreated controls (Figure 4D). An unbiased (i.e.

231 randomised and blinded) synchondrosis patency scoring system showed that the increased patency of the ISS

232 in the mutant mice treated on the frontal bone was statistically significant $(\mathrm{p}<0.05$; Figure $4 \mathbf{E})$. Histological

233 analysis confirmed that the ISS was abnormal in the mutant mice compared to WT while the SOS was normal

234 in both (Figure $\mathbf{4 G + H})$. The mutant ISS cartilage appears ectopic to the cranial base with a reduced number

235 of chondrocytes and is cone-shaped rather than rectangular. Frontal loaded mutant mice show normal ISS

236 cartilage comparable to the untreated WT mice (Figure 4I), but parietal loaded mice showed no difference to

237 untreated controls (Figure 4J). This data supports an interpretation that cranial loading has an effect on the cranial base, which in turn, contributes to the normalised skull shape, in particular skull length. 


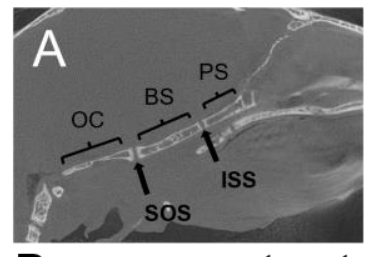

\section{B untreated}
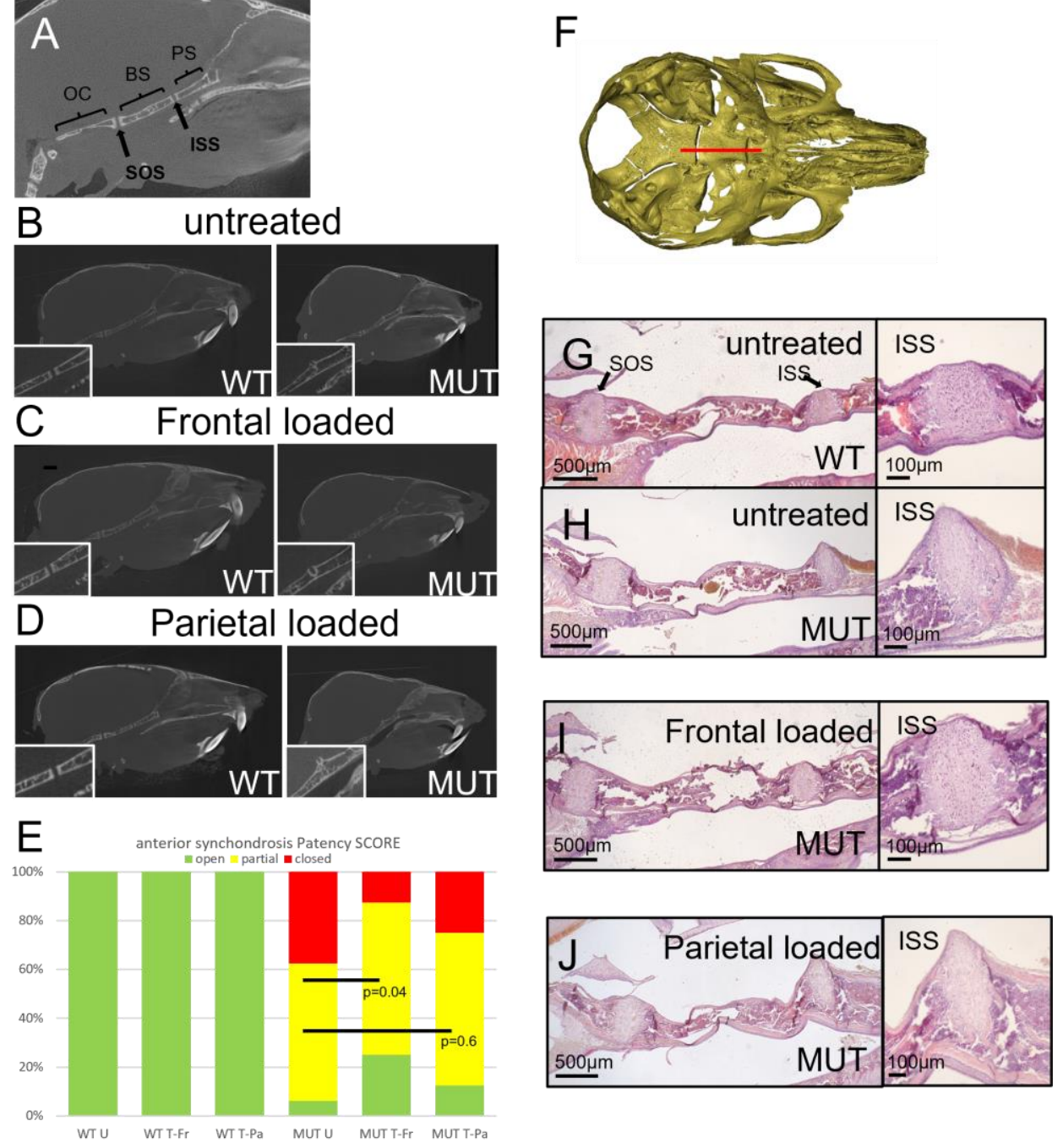

Figure 4

Changes in skull base morphology and histology following cranial bone loading.

A Schematic of the skull base showing anatomical structures. OC=occipital; BS=basi-sphenoid; PS=pre-sphenoid; SOS=spheno-occipital synchondrosis; ISS=intersphenoidal synchondrosis. B-D Micro-CT images of sagittal, midline sections through the skull. Untreated WT skulls show a fully patent ISS (inset) while MUT synchondroses have closed (B). Frontal loaded skulls show improved patency of the ISS (C), while parietal loaded skulls do not (D). E Patency of the anterior synchondrosis (ISS) was scored blinded for sample identity. While all WT synchondroses are open (green) at P21, the majority of the untreated MUT skull bases have a partially (yellow) or fully closed (red) ISS. Skulls loaded on the frontal bone show a statistically significant improvement while parietal loaded skulls do not. Untreated-WT (WT U) $\mathrm{n}=12$, frontal loaded-WT (WT T-Fr) $\mathrm{n}=15$, parietal loaded-WT (WT T-Pa) $\mathrm{n}=9$, untreated MUT (MUT U) $\mathrm{n}=16$, frontal loaded MUT (MUT T-Fr) n=7, parietal loaded MUT (MUT T-Pa) n=8. F Sagittal, midline sections through the skull base (red line) at P21 were stained with H\&E. G-J Histological analysis of the Crouzon-associated phenotypic features following cranial loading. G A wild-type skull base showing the bones seperated by sychondroses. SOS=sphenooccipital synchondrosis; ISS=intersphenoidal synchondrosis. The magnified image of the ISS shows a rectangular cartilage situated between endochodral bone. H Mutant (MUT) ISS shows an aberrant cone shape and the cartilage structure protrudes cranially. I Animals treated with frontal bone loading show a more normal synchondroses. J Animals treated with parietal bone loading show no improvement and look similar to untreated synchondroses. 


\section{Comparing the pattern of mechanical strain distribution across the skull between frontal and parietal}

\section{8 loading.}

259 To understand the pattern of mechanical strain distribution across the skull due to the loading at the frontal 260 and parietal, we used a previously validated finite element (FE) model ${ }^{30,31}$. The FE model was developed 261 based on a P3 WT mouse skull and was used to model calvarial growth. We use this model to predict the 262 shape and pattern of tissue differentiation at P7 and loaded the model at P7 analagous to our in vivo 263 experiments (Figure 5A). The pattern of first princial strain highlights that frontal loading induced a lower 264 level of strain across the coronal suture (see highlighted dash boxes in Figure 5B\&C) compared to parietal 265 loading. It also shows that the load applied to the skull roof induced a mechanical strain on the skull base. 266 Interestingly, the level of induced strain across the intersphenoidal synchondrosis (ISS) was lower in the 267 frontal loading compare to the parietal loading group (see highlighted dash boxes in Figure 5D\&E). 


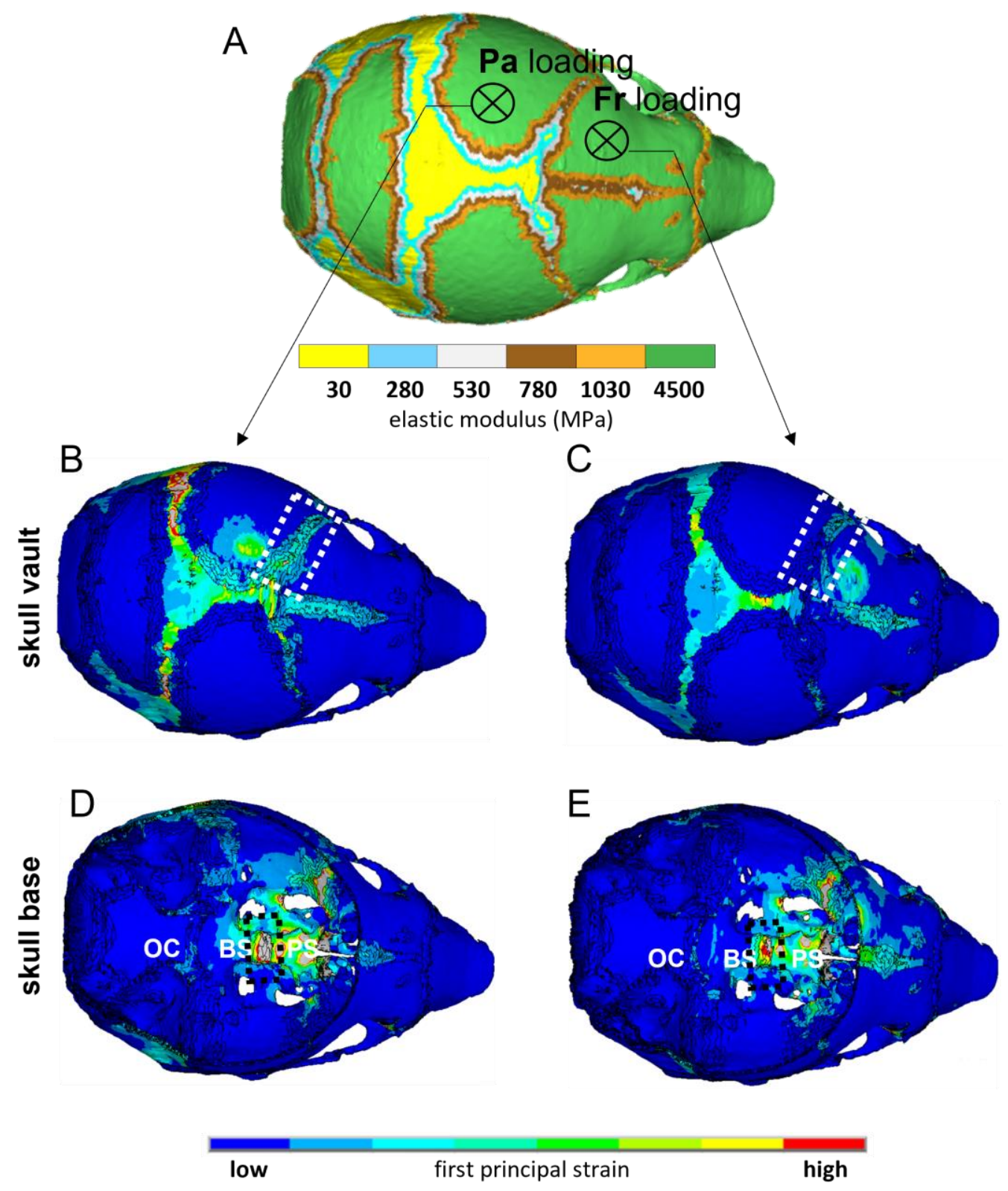

Figure 5 Comparing the pattern of mechanical strain distribution across the skull between frontal and

parietal loading. A Finite element model of a P3 mouse skull that was computationally grown to P7 and used to investigate the difference in the pattern of strain distribution due to the frontal and parietal loading. It indicates the mechanical properties of different skull regions and the anatomical position that were loaded analogous to the in vivo experiments. B-C Comparing the pattern of first principal strain across the skull roof between parietal and frontal loading respectively. The dashed box highlights the coronal suture. D-E Comparing the pattern of first principal strain across the skull base between parietal and frontal loading respectivly. The dashed box highlights the ISS (intersphenoidal synchondrosis). OC=occipital; BS=basi-sphenoid; PS=pre-sphenoid. 


\section{3. Discussion}

280 This study has shown that the application of external mechanical loading on the skull of a syndromic

281 craniosynostosis mouse model can prevent the complete closure of the coronal suture and of the anterior

282 synchondrosis, which in turn reduces the damaging effects on skull shape and size, partially restoring normal

283 skull appearance.

284 Accurate loading of the individual calvarial bones was achieved using a novel set up that was developed enabling us to apply force up to a predetermined maximum of 0.1 N. No adverse effects were observed in any of our loading procedures (total of 670) so we conclude this to be a safe approach for cranial loading in mouse. While the mice were subjected to general anaesthesia, this was mainly to avoid any movement during the procedure and we don't foresee this to be necessary when applied to patients. Further work will focus on the optimisation of the force/frequency needed to achieve the desired effect and on answering the question whether an initial loading treatment is sufficient to prevent complete synostosis or if loading needs to be applied long term to avoid the process of synostosis recommencing.

Overall we identified a significant effect on the size and shape of Crouzon skulls after frontal loading only.

293 This can be explained by the characteristic feature of the coronal suture, where the parietal bone partially 294 overlaps the frontal bone. Loading the frontal bone will load the soft sutural tissue under tension, that is likely to cause a certain level of shear stress. Cellular tension has been shown to activate the ROCK/TAZ pathway in vitro $^{32}$, but further in vivo analysis of the mechanotransduction effects on the sutural mesenchyme can potentially unravel the precise mechanism. While we anticipated the effect to be more pronounced on the loaded side (L) compared to the unloaded side (R), analysis showed only a small number of samples that presented with an asymmetric presentation of coronal synostosis rescue. A likely explanantion for this is that the mechanical strain that has been induced as a result of the unilateral loading transfers onto the unloaded coronal suture on the opposite side. The finite element data confirm that a low level of strain was indeed induced on the unloaded coronal suture albeit higher in the parietal loaded group compare to the frontal loaded group. At the same time, our observation that cranial loading prevents complete fusion of the synchondrosis supports the theory that loading the calvarial can impact the skull base through the 3D structural framework of the cranium. 
306 In our hands, the Crouzon Fgfr2-C342Y mouse model on a CD-1 background shows near complete fusion of 307 the coronal suture at P21, the end point of our experiments ${ }^{15,26}$. Compared to unloaded controls, frontal loaded sutures showed a mixed histological picture with increased patency on the one hand and reduction of the sutural mesenchyme on the other hand. Disappearance of the sutural mesenchyme by premature differentiation into osteoblast and bone is typical of the pathological synostosis process caused by FGFR2 mutation $^{33}$ and allows the fusion of the flanking bones. In this case however, daily application of mechanical tension prevents the fusion, while it seems unable to prevent the premature differentiation of the mesenchyme. As a result, the coronal suture retains it's patency allowing the skull to grow normally without the compensatory growth associated with coronal synostosis ${ }^{34}$.

An additional, unexpected discovery was that apart from partially rescueing the coronal suture synostosis phenotype, we were also able to partially rescue the premature closure of the anterior synchondrosis (i.e. intersphenoidal synchondrosis (ISS)) in the skull base of the Crouzon mouse. Prevention of premature fusion skull length that was observed in this study. Anatomically, the mouse ISS is similar to that in human and natural fusion of the human ISS occurs between 2-4 years of age ${ }^{35}$. There is no CT data available for the premature fusion of the ISS in human Crouzon syndrome patients, but in the Crouzon mouse model the ISS is fused prematurely at P21 as shown by by Liu et al. ${ }^{29}$ and this study. A significant delay in the premature fusion was identified in the frontal loaded Crouzon mice, which suggests that cranial loading transfers its effect indirectly to the synchondroses in the skull base. This was also corroborated by our finite element results that showed that calvarial loading induced mechanical strain on the skull base and ISS. Histologically, the mutant ISS displays as a cone shape cartilage, protruding rostrally from the sphenoidal flanking bones with a bony bridge forming caudally. We speculate that applying mechanical tension on the anterior side of the ISS (i.e. the presphenoidal bone) causes shear stress on the caudal side preventing the formation of the bony bridge and the subsequent protrusion of the cartilaginous synchondrosis. Alternatively, a mechanotransductional effect on the developing chondrocytes in the synchondrosis is possible, as it is known that the formation and fusion of the ISS is regulated by the Indian and Sonic Hedgehog pathways ${ }^{36}$. Clinically, skull base abnormalities are difficult to resolve surgically because of the anatomical position of the target 
333 tissue. External application of a corrective mechanical force may provide be a possible way to address this 334 clinical challenge.

335 It is clear that the proposed approach in this study can be further optimised and need to undergo a feasibility 336 study in a larger animal model in prepration for clinical translation if its efficacy is proven. At present it is 337 seems that such an approach can only be deployed on patients that present with sutures that are not fully fused 338 i.e. have not completed the synostosis process. The number of craniosynostosis patients that present with partially patent sutures is estimated to be 5-10\% at Great Ormond Street Hospital (D Dunaway 2021, personal 340 communiation). Another potential application of the cranial loading can be to prevent the healing of surgically 341 seperated calvarial bones post-operatively (aka resynostosis). This is an important clinical problem causing 342 significant morbidity leading to the need for repeated reoperations. Attempts to prevent reoperation have been 343 made using glypican-3 containing nanotubes to inhibit cranial bone regeneration in a Crouzon mouse model 344 and by regenerating a functional suture using Gli+ mesenchymal stem cells in a Twist1 mouse model of 345 craniosynostosis ${ }^{19,37}$. While these potential treatments have not been applied to patients yet, they will be 346 invasive procedures involving the introduction of pharmacological or cellular agents with potential unwanted 347 side effects. It may be possible to achieve a similar effect using cranial loading on patients post-surgery. 348 In summary, in vivo calvarial loading using pre-weaning Crouzon mice shows a distinct phenotypic effect 349 indicted by a normalised skull morphology and improved patency of the coronal suture and anterior 350 synchondrosis. Further studies into the use of mechanotransduction to delay or prevent pathogenic suture 351 closure have the potential to be a first important step towards the development of a non-invasive, non-surgical 352 treatment alternative for children with progressive craniosynostosis. 


\section{$354 \quad$ 4. Materials and methods} \\ 4.1. Animals}

Crouzon mouse model ( $F g f r 2^{\text {tm4Lni. }}$ aka $F g f r 2 c^{\mathrm{C} 342 \mathrm{Y}}$; MGI:3053095). $F g f r 2 c^{\mathrm{C} 342 \mathrm{Y}}$ were re-derived through the 357 European Mouse Mutant Archive (EMMA) at MRC Harwell as previously described ${ }^{26}$. All animal procedures were performed in accordance with the UK Animals (Scientific Procedures) Act 1986 (Project License number: 70/8817) and maintained by UCL Biological Services.

\subsection{Mechanical loading}

An experimental loading set up was developed to precisely load the cranial bones of mice in vivo. Here an actuator (T-LSR series, Zaber Technologies: res. $50 \mu \mathrm{m}$, max. load $200 \mathrm{~N}$ ) and a force sensor (GSO Series, Transducer Techniques: res. $0.01 \mathrm{~N}$ with $1 \mathrm{~N}$ capacity) were configured to a custom developed LabVIEW program. This portable set up enabled us to carry out precise cyclic loading of mouse skulls.

\subsection{Phenotypic analysis}

Animals were weighed using a fine balance. An electronic caliper (Fisher) was used to measure the crown-rump length and head length of the animal.

\subsection{Micro-CT analysis}

\subsubsection{Micro-CT scanning}

At the end of experiment, animals were killed by a schedule 1 method or a non-schedule 1 method (asphyxiated using rising concentrations of $\mathrm{CO} 2$ ). Then animals were fixed and stained with Alizarin Red to better visualise the calvarial bones and sutures. They were then micro CT scanned (X-Tek HMX 160, XTek 373 Systems Ltd, Tring, Herts., UK).

\section{$374 \quad$ 4.4.2. Image processing}

375 CT images were imported into an image processing software (Avizo Lite, FEI V9.2., Thermo Fisher

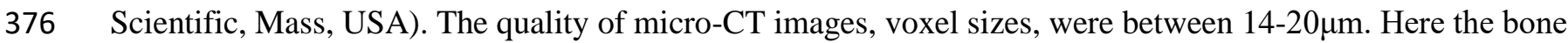
was segmented. Before segmentation micro-CT images were aligned in a similar position in different planes.

378 Following the alignment of the raw CT images, 3D surface volumes were reconstructed and a range of parameters were measured.

\subsubsection{Quantitative analysis}


381 In the lateral view, the differences in the length, height and the overall morphology of the calvarial were 382 observed between the groups. The dorsal view, highlights the suture fusion across the calvaria across the 383 groups. The skull length was measured in the sagittal plane between the most anterior-medial point of the nasal bone and the most posterior-medial point of the occipital bone. Skull width was then measured in the transverse plane between the most posterior-inferior point on the interparietal. Skull height was measured in the sagittal plane between the presphenoid and the most superior part of the skull, covering bulging area in the mutant types. In addition to the aforementioned measurements a number of other features of the skulls were measured. Landmarks were placed on the cranium on the boundaries between the bones of different regions of skull. This was to measure the dimensions of these bones individually in more details.

\subsubsection{Patency scoring of synchondroses.}

391 Sagittal sections through the midline of whole skull micro-CT images were presented to at least three observers blinded for treated and untreated groups. SOS and SIS synchondroses were scored as open, partially closed $(<50 \%$ of the cranial base height is filled with bone) or closed $(<50 \%$ of the cranial base height is filled with bone). Scores were averaged and plotted against expected frequencies in untreated groups.

\subsection{Fluorescent Alizarin Red staining}

Mice were injected intraperitoneally with 5ul/gram body weight of a $5 \mathrm{mg} / \mathrm{ml}$ Alizarin complexone (VWR

20118.081) made up in 2\% NaHCO3 in PBS. Calvaria were dissected 24 hours after injection and imaged

using a Zeiss upright fluorescent microscope with a 550nm filter.

\subsection{Histological analysis}

400 For paraffin embedding, mouse heads were skinned and fixed in 10\% Formalin overnight before graded 401 dehydration in ethanol. Embryos were cleared in analytical grade xylene (Fisher) before paraffin wax 402 displacement in a $60^{\circ} \mathrm{C}$ oven. The samples were embedded and sectioned between 8-10um on the sagittal 403 plane using a microtome (Leica). Sections were stained with Haematoxylin and Eosin.

\subsection{Statistical analysis}

SPSS Statistics 22 (IBM) software was used as the primary statistical package for data analysis. First, the data was tested for normality using Shapiro-Wilk test to determine the use of parametric or non-parametric tests. 
408 groups for quantification. $\mathrm{Chi}^{2}$-test was used for statistical analysis of the synchodrosis patency score. One409 way ANOVA with Tukey posthoc or non-parametric Kruskal Wallis with Dunn-Bonferroni posthoc test was 410 adopted for analysis of three or more groups. A p-value of $<0.05$ was considered significant.

\section{$411 \quad$ 4.8. Finite element analysis}

412 A previously validated finite element model of mouse skull growth was used here ${ }^{30,31}$. In brief, we 413 reconstructed micro-CT images of a P3 WT mouse (Avizo FEI Visualization Sciences Group, Merignac 414 Cedex, France) consisted of bone, sutures, and intracranial volume (ICV) that broadly represented the brain. 415 The whole model was then transformed into a 3D solid meshed model and imported to a finite element solver, 416 ANSYS v.18 (ANSYS Inc., Canonsburg, PA, USA). Isotropic (linear and elastic) material properties were 417 assigned to all sections with a thermal coefficient defined only for the ICV. Bone and suture were assumed to 418 have an elastic modulus of $3500 \mathrm{MPa}$ and $30 \mathrm{MPa}$ respectively at age $\mathrm{P} 3^{28,38}$. The elastic modulus of the ICV 419 was assumed to be $10 \mathrm{MPa}$. The bone and suture materials were assumed to have a Poisson's ratio of 0.3. The 420 ICV value was 0.48 . The bone-suture interfaces and bone-suture-intracranial volume interfaces were assumed 421 to be perfectly connected. Three nodes on the presphenoid bone were constrained in all degrees of freedom. 422 The presphenoid bone was constrained based on our previous examination of the growth of the WT mouse 423 skull that revealed that this bone grows centrically during development and can be considered to effectively 424 remain at the same position during the skull development ${ }^{30}$. The P3 model was then virtually grown to P7 (as 425 described in Marghoub et al. ${ }^{31}$ ) and at P7 was loaded at the fronal and parietal analogoun to our in vivo 426 experiment. The pattern of first principal strain across the skull were then compared between the two groups. 


\section{Competing interests}

The authors have no competing or financial interests to declare.

\section{Author contributions}

M.M., E.P. and A.A. conceived and designed the project; M.H., D.S., M.M., A.M., D.J. and E.P. acquired, analysed and interpreted data; E.P. and M.M. wrote the paper; all authors commented on and contributed to the paper.

\section{Acknowledgements}

The Fgfr $2 c^{\mathrm{C} 342 \mathrm{Y}}$ mouse colony was derived from the EMMA Consortium. This work was supported by Newlife, the charity for disabled children (award 17-18/18), the Royal Academy of Engineering (10216/119); and the Engineering and Physical Science Research Council (EP/R513143/1-176497) and University College London. EP is a GOSHCC Principal Investigator. This research was supported by the NIHR Great Ormond Street Hospital Biomedical Research Centre. The views expressed are those of the author(s) and not necessarily those of the NHS, the NIHR or the Department of Health. We are grateful to Michael Fagan for micro-CT scanning support and to Susan Herring for advice and support throughout this study. 


\section{References}

4291 Lajeunie, E., Le Merrer, M., Bonaiti-Pellie, C., Marchac, D. \& Renier, D. Genetic study of nonsyndromic coronal craniosynostosis. Am J Med Genet 55, 500-504, doi:10.1002/ajmg.1320550422 (1995). EUROCAT. European Platform on Rare Disease Registration-Prevalence, <https://eu-rdplatform.jrc.ec.europa.eu/eurocat/eurocat-data/prevalence_en> (2021).

Boyadjiev, S. A. \& International Craniosynostosis, C. Genetic analysis of non-syndromic craniosynostosis. Orthod Craniofac Res 10, 129-137, doi:10.1111/j.1601-6343.2007.00393.x (2007). from history to hydrogen bonds. Orthod Craniofac Res 10, 67-81, doi:10.1111/j.1601-6343.2007.00389.x (2007).

Wilkie, A. O. et al. Prevalence and complications of single-gene and chromosomal disorders in craniosynostosis. Pediatrics 126, e391-400, doi:10.1542/peds.2009-3491 (2010).

Twigg, S. R. \& Wilkie, A. O. A Genetic-Pathophysiological Framework for Craniosynostosis. Am J Hum Genet 97, 359-377, doi:10.1016/j.ajhg.2015.07.006 (2015).

Ornitz, D. M. \& Marie, P. J. Fibroblast growth factors in skeletal development. Current topics in developmental biology 133, 195-234, doi:10.1016/bs.ctdb.2018.11.020 (2019).

Sanchez-Lara, P. A. et al. Fetal constraint as a potential risk factor for craniosynostosis. Am J Med Genet A 152A, 394-400, doi:10.1002/ajmg.a.33246 (2010).

O'Hara, J. et al. Syndromic Craniosynostosis: Complexities of Clinical Care. Mol Syndromol 10, 83-97, doi:10.1159/000495739 (2019).

Sawh-Martinez, R. \& Steinbacher, D. M. Syndromic Craniosynostosis. Clin Plast Surg 46, 141-155, doi:10.1016/j.cps.2018.11.009 (2019).

11 Wang, J. C., Nagy, L. \& Demke, J. C. Syndromic Craniosynostosis. Facial Plast Surg Clin North Am 24, 531543, doi:10.1016/j.fsc.2016.06.008 (2016).

2 Goldstein, J. A., Paliga, J. T., Taylor, J. A. \& Bartlett, S. P. Complications in 54 frontofacial distraction procedures in patients with syndromic craniosynostosis. J Craniofac Surg 26, 124-128, doi:10.1097/SCS.0000000000001320 (2015).

Lee, K. K. L., Stanier, P. \& Pauws, E. Mouse Models of Syndromic Craniosynostosis. Mol Syndromol 10, 5873, doi:10.1159/000491004 (2019). 
45714 Shukla, V., Coumoul, X., Wang, R. H., Kim, H. S. \& Deng, C. X. RNA interference and inhibition of MEKERK signaling prevent abnormal skeletal phenotypes in a mouse model of craniosynostosis. Nat Genet 39, 1145-1150, doi:10.1038/ng2096 (2007).

46015 Lee, K. K. L. et al. Overexpression of Fgfr2c causes craniofacial bone hypoplasia and ameliorates craniosynostosis in the Crouzon mouse. Dis Model Mech 11, doi:10.1242/dmm.035311 (2018).

Shin, H. R. et al. PIN1 is a new therapeutic target of craniosynostosis. Hum Mol Genet 27, 3827-3839, doi:10.1093/hmg/ddy252 (2018).

Seda, M. et al. An FDA-Approved Drug Screen for Compounds Influencing Craniofacial Skeletal Development and Craniosynostosis. Mol Syndromol 10, 98-114, doi:10.1159/000491567 (2019). e0234073, doi:10.1371/journal.pone.0234073 (2020). 184, 243-256 e218, doi:10.1016/j.cell.2020.11.037 (2021). Histopathol 32, 751-760, doi:10.14670/HH-11-858 (2017). 
26 Peskett, E. et al. Analysis of the Fgfr2C342Y mouse model shows condensation defects due to misregulation of Sox9 expression in prechondrocytic mesenchyme. Biol. Open 6, 223-231, doi:bio.022178 [pii];10.1242/bio.022178 [doi] (2017).

Dudakovic, A., Nam, H. K., van Wijnen, A. J. \& Hatch, N. E. Genetic background dependent modifiers of craniosynostosis severity. J Struct Biol 212, 107629, doi:10.1016/j.jsb.2020.107629 (2020).

Moazen, M., Peskett, E., Babbs, C., Pauws, E. \& Fagan, M. J. Mechanical properties of calvarial bones in a mouse model for craniosynostosis. PLoS One 10, e0125757, doi:10.1371/journal.pone.0125757 (2015). FGFR2(C342Y) mutation are cranial bone-dependent. Calcif. Tissue Int 92, 451-466, doi:10.1007/s00223-0139701-2 [doi] (2013).

Marghoub, A. et al. Characterizing and Modeling Bone Formation during Mouse Calvarial Development. Phys Rev Lett 122, 048103, doi:10.1103/PhysRevLett.122.048103 (2019). Marghoub, A. et al. Predicting calvarial growth in normal and craniosynostotic mice using a computational approach. J Anat 232, 440-448, doi:10.1111/joa.12764 (2018).

Li, W. et al. ROCK-TAZ signaling axis regulates mechanical tension-induced osteogenic differentiation of rat cranial sagittal suture mesenchymal stem cells. J Cell Physiol 235, 5972-5984, doi:10.1002/jcp.29522 (2020).

Holmes, G. et al. Early onset of craniosynostosis in an Apert mouse model reveals critical features of this pathology. Dev Biol 328, 273-284, doi:10.1016/j.ydbio.2009.01.026 (2009).

Kreiborg, S. et al. Comparative three-dimensional analysis of CT-scans of the calvaria and cranial base in Apert and Crouzon syndromes. J Craniomaxillofac Surg 21, 181-188, doi:10.1016/s1010-5182(05)80478-0 (1993).

Madeline, L. A. \& Elster, A. D. Suture closure in the human chondrocranium: CT assessment. Radiology 196, 747-756, doi:10.1148/radiology.196.3.7644639 (1995).

Young, B. et al. Indian and sonic hedgehogs regulate synchondrosis growth plate and cranial base development and function. Dev Biol 299, 272-282, doi:10.1016/j.ydbio.2006.07.028 (2006).

7 Bariana, M., Kaidonis, J. A., Losic, D., Ranjitkar, S. \& Anderson, P. J. Titania nanotube-based protein delivery system to inhibit cranial bone regeneration in Crouzon model of craniosynostosis. Int J Nanomedicine 14, 63136324, doi:10.2147/IJN.S202090 (2019).

38 Henderson, J. H., Chang, L. Y., Song, H. M., Longaker, M. T. \& Carter, D. R. Age-dependent properties and quasi-static strain in the rat sagittal suture. J Biomech 38, 2294-2301, doi:10.1016/j.jbiomech.2004.07.037 (2005). 


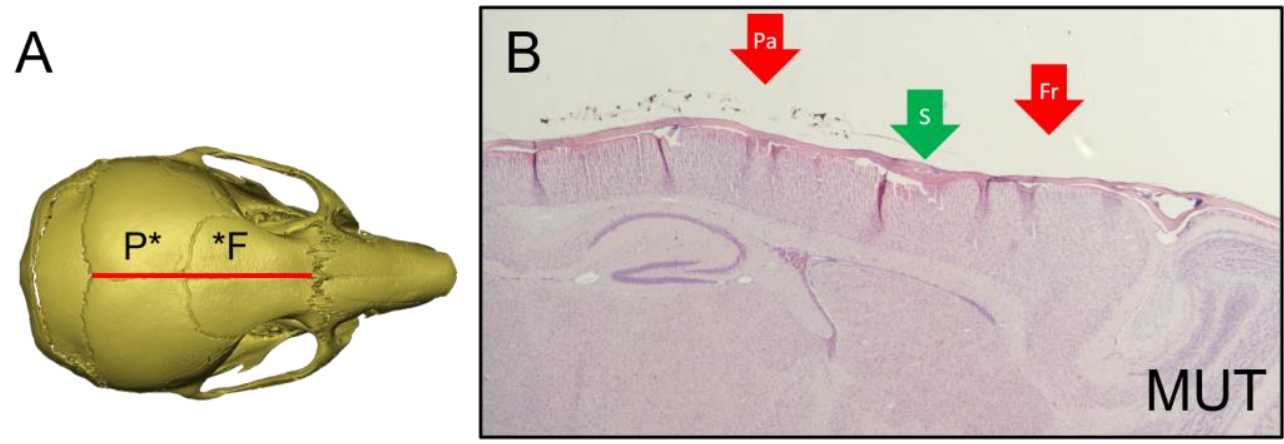

\section{untreated}

Parietal loaded

\section{Frontal loaded}
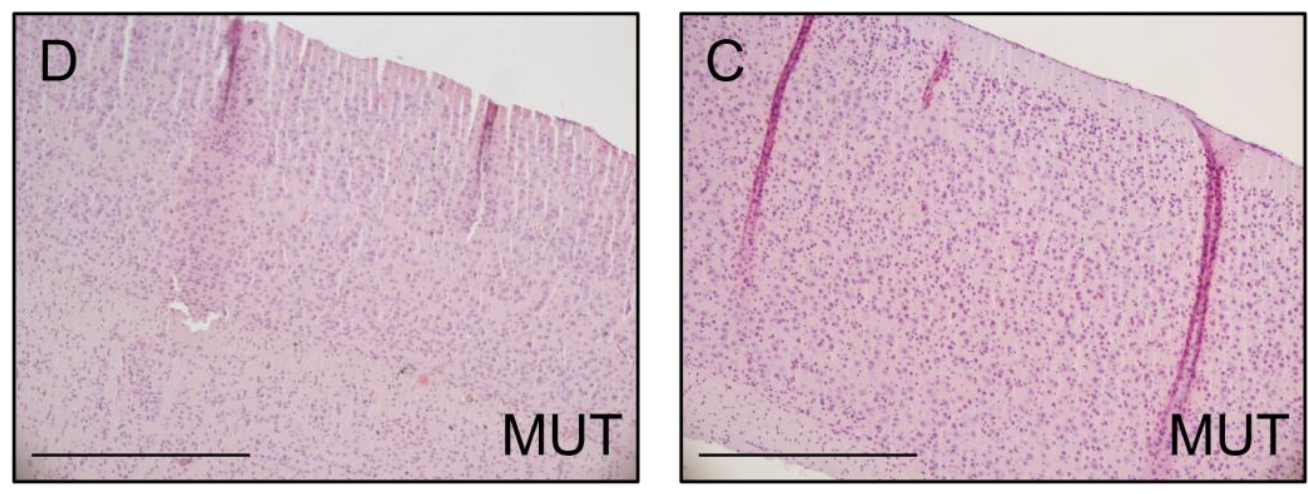

Supplementary Figure 1 Histological analysis of brain tissue following cranial bone loading. A Sagittal, midline sections through the brain (red line) at P21 were stained with H\&E. B Low magnification image of a mutant brain showing the fused coronal suture (green arrow, $\mathrm{S}$ ) as well as the approximate location of frontal (red arrow, Fr) and parietal (red arrow, Pa) loading. C High magnification image of a frontal loaded mutant brain showing normal cortical lamination. D High magnification image of a parietal loaded mutant brain showing normal cortical lamination. 
524 Supplementary Video 1a Video footage (25s) of cranial bone loading treatment protocol. This video shows a P7 WT

525 mouse being loaded on the frontal bone.

526

527 Supplementary Video 1b Video footage (25s) of cranial bone loading treatment protocol. This video shows a P14 WT 528 mouse being loaded on the frontal bone.

530 Supplementary Video 2a Video footage (52s) showing behaviour of three WT P21 litter mates (L19, L20 and L21) after 531 completing the two-week cranial bone loading treatment protocol.

532

533 Supplementary Video 2b Video footage showing behaviour of three MUT P21 litter mates (L18, L25 and L26) after 534 completing the two-week cranial boneloading treatment protocol. 